\title{
LMI Conditions for Robust Stability of 2D Linear Discrete-Time Systems
}

\author{
A. Hmamed, ${ }^{1}$ M. Alfidi, ${ }^{1}$ A. Benzaouia, ${ }^{2}$ and F. Tadeo $^{3}$ \\ ${ }^{1}$ Laboratoire d'Electronique, Signaux, Systemes et Informatique (LESSI), \\ Département de Physique, Faculté des Sciences Dhar El Mehraz, B.P. 1796, 30000 Fes-Atlas, Morocco \\ ${ }^{2}$ Research Unit, Constrained and Robust Regulation, Department of Physics, \\ Faculty of Sciences Semlalia, B.P. 2390, 40000 Marrakech, Morocco \\ ${ }^{3}$ Departamento de Ingenieria de Sistemas y Automática, Universidad de Valladolid, \\ 47005 Valladolid, Spain
}

Correspondence should be addressed to F. Tadeo, fernando@autom.uva.es

Received 11 May 2007; Revised 1 October 2007; Accepted 8 February 2008

Recommended by Pramod Khargonekar

Robust stability conditions are derived for uncertain 2D linear discrete-time systems, described by Fornasini-Marchesini second models with polytopic uncertainty. Robust stability is guaranteed by the existence of a parameter-dependent Lyapunov function obtained from the feasibility of a set of linear matrix inequalities, formulated at the vertices of the uncertainty polytope. Several examples are presented to illustrate the results.

Copyright (C) 2008 A. Hmamed et al. This is an open access article distributed under the Creative Commons Attribution License, which permits unrestricted use, distribution, and reproduction in any medium, provided the original work is properly cited.

\section{Introduction}

Robust stability is an important issue for many applications, as it is the first requirement for any design. Methodologies to check stability are usually derived from Lyapunov functions. In particular, quadratic stability (QS) has played a central role, as it gives conditions simple to check. In fact, during the last decades, the QS concept has been widely used for robust analysis and control design for uncertain systems [1-4]. Using QS, the stability of a polytope of matrices can be checked by testing the feasibility of a set of linear matrix inequalities (LMIs). An important characteristic is that this set of LMIs involves only the vertices of the uncertain domain, which simplifies the robust stability tests [3].

Unfortunately, it is known that tests derived from QS might lead to very conservative results in several cases. Recently, other techniques have appeared, based on parameterdependent Lyapunov functions [5-8] or piecewise Lyapunov functions [9, 10], providing less conservative results. In particular, [5] gives sufficient LMI conditions for robust stability of uncertain discrete-time systems. The generalization of these conditions to robust D-stability has 
been published in [11]. The key idea used in these papers, which will be used here, is to introduce new variables and increase the size of the LMIs, to obtain sufficient conditions for the existence of a parameter-dependent Lyapunov function.

This paper concentrates on two-dimensional (2D) systems because these 2D systems play important roles in image data processing and transformation, water stream heating, thermal processes, biomedical imaging, gas absorption, and many other areas [12], so the study of these systems has received much attention in past decades. Thus, a great number of stability and control results, related to 2D systems, have been reported in the literature, for example, using 2-D Lyapunov equations, some stability results have already been obtained in [13-16].

In particular, the problem of stability for uncertain 2D discrete-time systems is studied here. The class of systems under consideration is described by Fornasini-Marchesini models with polytopic uncertainty, which is frequent in image processing problems. However, it must be pointed out that the results are quite general, in the sense that with adequate modifications, parallel results can be obtained for other 2D systems (e.g., Roesser models, as presented in [17]). New sufficient conditions for robust stability are obtained from the feasibility of a set of LMIs formulated at the vertices of uncertainty polytope, extending the results proposed by the authors in [18]. Several examples will be presented and discussed to illustrate the results.

\section{Notation}

For real symmetric matrices $X$ and $Y$, the notation $X \geq Y$ (resp., $X>Y$ ) means that the matrix $X-Y$ is positive semidefinite (resp., positive definite). $I$ is the identity matrix with appropriate dimensions. $\|\cdot\|$ means the Euclidean norm. If no explicitly stated, matrices are assumed to have compatible dimensions. For symmetric matrices, off-diagonal blocks are abbreviated with " $*$ " as follows:

$$
\left[\begin{array}{ll}
X_{11} & X_{12} \\
X_{12}^{T} & X_{22}
\end{array}\right]=\left[\begin{array}{cc}
X_{11} & X_{12} \\
* & X_{22}
\end{array}\right] .
$$

\section{Problem formulation and preliminary results}

Consider the following 2D linear discrete-time system described by a Fornasini-Marchesini second model [16]

$$
x(i+1, j+1)=A\left[\begin{array}{l}
x(i+1, j) \\
x(i, j+1)
\end{array}\right]
$$

where $x(i, j) \in R^{n}$ is the state vector, $A \in R^{2 n \times n}$ is the dynamic matrix, and the boundary conditions are given by

$$
x(i, 0)=x_{0}(i), \quad x(0, j)=x_{0}(j), \quad i, j=0,1,2, \ldots
$$

We first introduce the notion of asymptotic stability of 2D discrete-time systems. 
Definition 2.1 (see [12]). The 2D linear discrete-time system (2.1) is said to be asymptotically stable if

$$
\lim _{k \rightarrow \infty}\|X(k)\|=0
$$

under $\sup _{j}\|X(0)\|<\infty$, where $X(k)=\{x(i, j): i+j=k\}$ and $\|X(k)\|=\sup _{x \in X(k)}\|x\|$.

The following lemma gives a sufficient condition for the asymptotic stability of 2D linear discrete-time systems described by (2.1) in terms of an LMI.

Lemma 2.2 (see [13]). The 2D linear discrete-time system (2.1) is asymptotically stable if there exist matrices $P_{11}>0, P_{22}>0, P_{12}=P_{12}^{T} \geq 0$, and $\Pi>0$ such that the following LMI holds:

$$
\left[\begin{array}{cc}
-\Pi & A^{T}\left(P_{11}+2 P_{12}+P_{22}\right) \\
* & -\left(P_{11}+2 P_{12}+P_{22}\right)
\end{array}\right]<0,
$$

where $\Pi=\left[\begin{array}{ll}P_{11} & P_{12} \\ P_{12}^{T} & P_{22}\end{array}\right]$.

Remark 2.3. As proven in [13], (2.4) includes the previous results in [14, 15].

Remark 2.4. The LMI (2.4) is useful for stability analysis but not for synthesis of controllers because it involves three variables $P_{11}, P_{12}$, and $P_{22}$ that render the linearization of the problem a difficult task. Thus, for the synthesis problem, the following alternative result will be used.

Lemma 2.5. The $2 D$ linear discrete-time system (2.1) is asymptotically stable if there exist matrices $P>0, Q>0, R=R^{T} \geq 0$, and $\Pi>0$ such that the following $L M I$ holds

$$
A^{T} P A-\Pi<0,
$$

where $\Pi=\left[\begin{array}{cc}P-Q-2 R & R \\ R^{T} & Q\end{array}\right]$, or equivalently

$$
\left[A^{T} A^{T}\right] \Pi\left[\begin{array}{l}
A \\
A
\end{array}\right]-\Pi<0 .
$$

Proof. It is possible to obtain (2.5) substituting $P_{11}=P-Q-2 R, P_{12}=R$, and $P_{22}=Q$ in (2.4) and using a Schur complement argument.

Suppose now that $A$ is not exactly known, but belongs to a given polytopic uncertain domain $\Omega$. In this way, any matrix inside the domain $\Omega$ can be written as a convex combination of the vertices $A_{i}$ of the uncertainty polytope:

$$
\Omega=\left\{A(\alpha): A(\alpha)=\sum_{i=1}^{N} \alpha_{i} A_{i}, \sum_{i=1}^{N} \alpha_{i}=1, \alpha_{i}>0\right\} .
$$

We begin our discussion by defining Robust Stability of system (2.1) under the structured model (2.7).

Definition 2.6. System (2.1) is robustly stable in the uncertainty domain (2.7) if there exist $P(\alpha)>0, Q(\alpha)>0$, and $R(\alpha) \geq 0$ such that

$$
A^{T}(\alpha) P(\alpha) A(\alpha)-\Pi(\alpha)<0
$$


for all $\alpha$ such that $A(\alpha) \in \Omega$, where

$$
\Pi(\alpha)=\left[\begin{array}{cc}
P(\alpha)-Q(\alpha)-2 R(\alpha) & R(\alpha) \\
R^{T}(\alpha) & Q(\alpha)
\end{array}\right]>0 .
$$

To the authors knowledge, there is no general and systematic way to formally determine $\Pi(\alpha)$ as a function of the uncertain parameter $\alpha$. Such a matrix $\Pi(\alpha)$ is called a parameterdependent Lyapunov matrix.

An effective way of addressing such problem is to look for a single Lyapunov matrix $\Pi(\alpha)=\Pi$ which solves inequality (2.8). Unfortunately, this approach is known to provide quite conservative results, but it constitutes one of the first results in the quadratic approach. The test for this kind of stability, also known as a quadratic stability (QS) test, is summarized in the following lemma.

Lemma 2.7. The uncertain system (2.1) is robustly stable in the uncertainty domain (2.7) if there exist matrices $P>0, Q>0, R=R^{T} \geq 0$, and $\Pi>0$ such that

$$
A_{i}^{T} P A_{i}-\Pi<0
$$

for all $i=1, \ldots, N$.

Theorem 2.8. The following conditions are equivalent.

(i) There exist matrices $P>0, Q>0, R=R^{T} \geq 0$, and $\Pi>0$ such that

$$
A^{T} P A-\Pi<0 .
$$

(ii) There exist matrices $P>0, Q>0, R=R^{T} \geq 0, \Pi>0, F \in R^{2 n \times n}$, and $G \in R^{n \times n}$ such that

$$
\left[\begin{array}{cc}
-\Pi+F A+A^{T} F^{T} & -F+A^{T} G^{T} \\
* & P-G-G^{T}
\end{array}\right]<0 .
$$

Proof. The proof is rather straightforward. First, if (2.10) holds for some $\Pi>0$, by setting $F=0$, $G=G^{T}=P$, and applying the Schur complement, (2.12) is satisfied. On the other hand, if (2.12) holds for some $(\Pi, F, G)$, multiplying $(2.12)$ from the left by $\Gamma^{T}$ and from the right by $\Gamma$, where $\Gamma^{T}=\left[I A^{T}\right]$, then (2.10) follows.

Remark 2.9. Condition (ii) appears as a direct expansion of condition (i) via its "Schur complement" formulation. An LMI is obtained in which the Lyapunov matrix $\Pi$ is not involved in any product with the dynamic matrix $A$, thanks to the introduction of the additional matrices $F$ and $G$. This feature enables one to write new robust stability conditions which, although sufficient, are not too conservative, as shown later in some numerical examples, thanks to the presence of the extra degrees of freedom provided by the introduction of matrices $F$ and $G$. 
The next three lemmas state sufficient conditions for the existence of a parameterdependent Lyapunov function $\Pi(\alpha)=\Pi^{T}(\alpha)>0$ given by

$$
\Pi(\alpha)=\sum_{i=1}^{N} \alpha_{i} \Pi_{i}=\sum_{i=1}^{N} \alpha_{i}\left[\begin{array}{cc}
P_{i}-Q_{i}-2 R_{i} & R_{i} \\
R_{i}^{T} & Q_{i}
\end{array}\right], \quad \text { where } \alpha_{i} \geq 0, i=1, \ldots, N, \quad \sum_{i=1}^{N} \alpha_{i}=1,
$$

such that (2.8) holds.

Lemma 2.10 (see [19]). The uncertain system (2.1) is robustly stable in the uncertainty domain (2.7) if there exist matrices $P_{i}>0, Q_{i}>0, R_{i}=R_{i}^{T} \geq 0$, and $\Pi_{i}=\left[\begin{array}{cc}P_{i}-Q_{i}-2 R_{i} R_{i} \\ R_{i}^{T} & Q_{i}\end{array}\right]>0, i=1, \ldots, N$ such that

$$
\begin{aligned}
& A_{i}^{T} P_{i} A_{i}-\Pi_{i}<-I, \\
& A_{i}^{T} P_{i} A_{j}+A_{j}^{T} P_{i} A_{i}+A_{i}^{T} P_{j} A_{i}-2 \Pi_{i}-\Pi_{j}<\frac{1}{(N-1)^{2}} I, \quad j=1, \ldots, N, j \neq i, \\
& A_{j}^{T} P_{i} A_{k}+A_{k}^{T} P_{i} A_{j}+A_{i}^{T} P_{j} A_{k}+A_{k}^{T} P_{j} A_{i}+A_{i}^{T} P_{k} A_{j}+A_{j}^{T} P_{k} A_{i}-2\left(\Pi_{i}+\Pi_{j}+\Pi_{k}\right) \\
& \quad<\frac{6}{(N-1)^{2}} I, \quad i=1, \ldots, N-2, j=i+1, \ldots, N-1, k=j+1, \ldots, N,
\end{aligned}
$$

then (2.8) holds with $\Pi(\alpha)=\Pi^{T}(\alpha)>0$ given by (2.13).

Lemma 2.11 (see [19]). The uncertain system (2.1) is robustly stable in the uncertainty domain (2.7) if there exist matrices $P_{i}>0, Q_{i}>0, R_{i}=R_{i}^{T} \geq 0, \Pi_{i}>0, F$, and $G$, for $i=1, \ldots, N$ such that

$$
\left[\begin{array}{cc}
-\Pi_{i}+F A_{i}+A_{i}^{T} F^{T} & -F+A_{i}^{T} G^{T} \\
* & P_{i}-G_{i}-G_{i}^{T}
\end{array}\right]<0,
$$

then (2.8) is verified with $\Pi(\alpha)>0$ given by (2.13).

Lemma 2.12 (see [19]). The uncertain system (2.1) is robustly stable in the uncertainty domain (2.7) if there exist matrices $P_{i}>0, Q_{i}>0, R_{i}=R_{i}^{T} \geq 0, \Pi_{i}>0, F_{i}$, and $G_{i}$ for $i=1, \ldots, N$ such that

$$
\begin{aligned}
& M_{i} \triangleq\left[\begin{array}{cr}
-\Pi_{i}+F_{i} A_{i}+A_{i}^{T} F_{i}^{T} & -F_{i}+A_{i}^{T} G_{i}^{T} \\
* & P_{i}-G_{i}-G_{i}^{T}
\end{array}\right]<-I, \\
& M_{i j} \triangleq\left[\begin{array}{cc}
\Gamma_{11} & \Gamma_{12} \\
* & \Gamma_{22}
\end{array}\right]<\frac{1}{(N-1)^{2}} I, \quad j=1, \ldots, N, j \neq i,
\end{aligned}
$$

where

$$
\begin{aligned}
& \Gamma_{11}=-\left(2 \Pi_{i}+\Pi_{j}\right)+F_{i} A_{j}+F_{j} A_{i}+F_{i} A_{i}+A_{j}^{T} F_{i}^{T}+A_{j}^{T} F_{i}^{T}+A_{i}^{T} F_{i}^{T}, \\
& \Gamma_{12}=-\left(2 F_{i}+F_{j}\right)+A_{i}^{T} G_{j}^{T}+A_{j}^{T} G_{i}^{T}+A_{i}^{T} G_{i}^{T}, \\
& \Gamma_{22}=2 P_{i}+P_{j}-\left(2 G_{i}+G_{j}\right)-\left(2 G_{i}+G_{j}\right)^{T}, \\
& M_{i j k} \triangleq\left[\begin{array}{cc}
\Upsilon_{11} & \Upsilon_{12} \\
* & \Upsilon_{22}
\end{array}\right]<\frac{6}{(N-1)^{2}} I, \quad i=1, \ldots, N-2, j=i+1, \ldots, N-1, k=j+1, \ldots, N,
\end{aligned}
$$


where

$$
\begin{aligned}
\Upsilon_{11}= & -2\left(\Pi_{i}+\Pi_{j}+\Pi_{k}\right)+\left(F_{i}+F_{k}\right) A_{j}+\left(F_{i}+F_{j}\right) A_{k}+\left(F_{j}+F_{k}\right) A_{i} \\
& +A_{j}^{T}\left(F_{i}+F_{k}\right)^{T}+A_{k}^{T}\left(F_{i}+F_{j}\right)^{T}+A_{i}^{T}\left(F_{j}+F_{k}\right)^{T}, \\
\Upsilon_{12}= & -2\left(F_{i}+F_{j}+F_{k}\right)+A_{j}^{T}\left(G_{i}+G_{k}\right)^{T}+A_{k}^{T}\left(G_{i}+G_{j}\right)^{T}+A_{i}^{T}\left(G_{j}+G_{k}\right)^{T}, \\
\Upsilon_{22}= & 2\left(P_{i}+P_{j}+P_{k}\right)-2\left(G_{i}+G_{j}+G_{k}\right)-2\left(G_{i}+G_{j}+G_{k}\right)^{T} .
\end{aligned}
$$

Remark 2.13. It is easy to see that the LMI conditions of Lemmas 2.10, 2.11, and 2.12 are less conservative than those provided in Lemma 2.7. For example, condition (2.14) contains (2.8) as a particular case when $P_{1}=P_{2}=\cdots=P_{N}=P$. This fact can be verified as follows. First, note that in the quadratic stability case, (2.14) reduces to (2.8). Second, computing the quadratic form with $P>0$ and $j \neq k$, it is possible to see that $\left(A_{j}-A_{k}\right)^{T} P\left(A_{j}-A_{k}\right) \geq 0$. Thus, one gets $A_{j}^{T} P A_{k}+A_{k}^{T} P A_{j} \leq A_{j}^{T} P A_{j}+A_{k}^{T} P A_{k}$, implying, in this particular case, that the LMIs (2.15) and (2.15) are always feasible.

\section{Main results}

In the following, we propose an LMI-based condition for finding a parameter-dependent Lyapunov matrix $\Pi(\alpha)>0$ satisfying (2.8) for every $A(\alpha) \in \Omega$, which is even less conservative than the results stated in the previous lemmas.

Theorem 3.1. The uncertain system (2.1) is robustly stable in the uncertainty domain (2.7) if there exist matrices $P_{i}>0, Q_{i}>0, R_{i}=R_{i}^{T} \geq 0, Y_{i i i}(1 \leq i \leq N), Y_{i i j}=Y_{j i i}^{T} Y_{i j i}(1 \leq i, j \leq N$, $i \neq j), Y_{i j l}=Y_{l j i^{\prime}}^{T}, Y_{j i l}=Y_{l i j}^{T}(1 \leq i<j<k \leq N)$, and $\Pi_{i}=\left[\begin{array}{cc}P_{i}-Q_{i}-2 R_{i} R_{i} \\ R_{i}^{T} & Q_{i}\end{array}\right]>0,(1 \leq i \leq N)$ such that

$$
\begin{aligned}
& A_{i}^{T} P_{i} A_{i}-\Pi_{i}<Y_{i i i}, \quad 1 \leq i \leq N, \\
& A_{i}^{T} P_{i} A_{j}+A_{j}^{T} P_{i} A_{i}+A_{i}^{T} P_{j} A_{i}-2 \Pi_{i}-\Pi_{j} \leq Y_{i i j}+Y_{i j i}+Y_{i i j \prime}^{T} \quad 1 \leq i, j \leq N, i \neq j, \\
& A_{j}^{T} P_{i} A_{k}+A_{k}^{T} P_{i} A_{j}+A_{i}^{T} P_{j} A_{k}+A_{k}^{T} P_{j} A_{i}+A_{i}^{T} P_{k} A_{j}+A_{j}^{T} P_{k} A_{i}-2\left(\Pi_{i}+\Pi_{j}+\Pi_{k}\right) \\
& \quad \leq Y_{i j k}+Y_{i k j}+Y_{j i k}+Y_{i j k}^{T}+Y_{i k j}^{T}+Y_{j i k \prime}^{T} \quad 1 \leq i<j<k \leq N, \\
& {\left[\begin{array}{cccc}
Y_{1 i 1} & Y_{1 i 2} & \cdots & Y_{1 i N} \\
Y_{2 i 1} & Y_{2 i 2} & \cdots & Y_{2 i N} \\
\vdots & \vdots & \ddots & \vdots \\
Y_{N i 1} & Y_{N i 2} & \cdots & Y_{N i N}
\end{array}\right] \leq 0, \quad 1 \leq i \leq N ;}
\end{aligned}
$$

then, for any $A(\alpha) \in \Omega$, a parameter-dependent matrix $\Pi(\alpha)>0$ given by (2.13) satisfies (2.8). 
Proof. It is clear that $\Pi(\alpha)$ given by $(2.13)$ is a positive-definite parameter Lyapunov matrix. Using (2.13), the definition of $A(\alpha) \in \Omega$ given in (2.7), and the direct expansion, one gets

$$
\begin{aligned}
& A(\alpha) P(\alpha) A(\alpha)-\Pi(\alpha) \\
= & A(\alpha) P(\alpha) A(\alpha)-\left(\sum_{i=1}^{N} \alpha_{i}\right)^{2} P(\alpha) \\
= & \sum_{i=1}^{N} \alpha_{i}^{3}\left(A_{i}^{T} P_{i} A_{i}-\Pi_{i}\right)+\sum_{i=1}^{N} \sum_{j=1, j \neq i}^{N} \alpha_{i}^{2} \alpha_{j}\left(A_{i}^{T} P_{i} A_{j}+A_{j}^{T} P_{i} A_{i}+A_{i}^{T} P_{j} A_{i}-2 \Pi_{i}-\Pi_{i}\right) \\
& +\sum_{i=1}^{N-2} \sum_{j=i+1}^{N-1} \sum_{k=j+1}^{N} \alpha_{i} \alpha_{j} \alpha_{k}\left(A_{i}^{T} P_{j} A_{k}+A_{i}^{T} P_{k} A_{j}+A_{j}^{T} P_{i} A_{k}+A_{k}^{T} P_{j} A_{i}+A_{j}^{T} P_{k} A_{i}+A_{k}^{T} P_{i} A_{j}-2\left(\Pi_{i}+\Pi_{j}+\Pi_{k}\right)\right) .
\end{aligned}
$$

Such an expansion is also adopted in [9]. Imposing conditions (3.1)-(3.4), one gets

$$
\begin{aligned}
& A(\alpha) P(\alpha) A(\alpha)-\Pi(\alpha) \\
& <\sum_{i=1}^{N} \alpha_{i}^{3} Y_{i i i}+\sum_{i=1}^{N} \sum_{j=1, j \neq i}^{N} \alpha_{i}^{2} \alpha_{j}\left(Y_{i i j}+Y_{i j i}+Y_{i i j}^{T}\right)+\sum_{i=1}^{N-2} \sum_{j=i+1}^{N-1} \sum_{k=j+1}^{N} \alpha_{i} \alpha_{j} \alpha_{k}\left(Y_{i j k}+Y_{i k j}+Y_{j i k}+Y_{i j k}^{T}+Y_{i k j}^{T}+Y_{j i k}^{T}\right) \\
& =\alpha_{1}\left[\begin{array}{c}
\alpha_{1} I \\
\alpha_{2} I \\
\vdots \\
\alpha_{N} I
\end{array}\right]^{T}\left[\begin{array}{cccc}
Y_{111} & \Upsilon_{112} & \cdots & \Upsilon_{11 N} \\
Y_{211} & Y_{212} & \cdots & Y_{21 N} \\
\vdots & \vdots & \ddots & \vdots \\
Y_{N 11} & Y_{N 12} & \cdots & Y_{N 1 N}
\end{array}\right]\left[\begin{array}{c}
\alpha_{1} I \\
\alpha_{2} I \\
\vdots \\
\alpha_{N} I
\end{array}\right]+\alpha_{2}\left[\begin{array}{c}
\alpha_{1} I \\
\alpha_{2} I \\
\vdots \\
\alpha_{N} I
\end{array}\right]^{T}\left[\begin{array}{cccc}
Y_{121} & Y_{122} & \cdots & \Upsilon_{12 N} \\
Y_{221} & Y_{222} & \cdots & Y_{22 N} \\
\vdots & \vdots & \ddots & \vdots \\
Y_{N 21} & Y_{N 22} & \cdots & Y_{N 2 N}
\end{array}\right]\left[\begin{array}{c}
\alpha_{1} I \\
\alpha_{2} I \\
\vdots \\
\alpha_{N} I
\end{array}\right] \\
& +\cdots+\alpha_{N}\left[\begin{array}{c}
\alpha_{1} I \\
\alpha_{2} I \\
\vdots \\
\alpha_{N} I
\end{array}\right]^{T}\left[\begin{array}{cccc}
\Upsilon_{1 N 1} & \Upsilon_{1 N 2} & \cdots & \Upsilon_{1 N N} \\
Y_{2 N 1} & \Upsilon_{2 N 2} & \cdots & \Upsilon_{2 N N} \\
\vdots & \vdots & \ddots & \vdots \\
Y_{N N 1} & \Upsilon_{N N 2} & \cdots & \Upsilon_{N N N}
\end{array}\right]\left[\begin{array}{c}
\alpha_{1} I \\
\alpha_{2} I \\
\vdots \\
\alpha_{N} I
\end{array}\right] \\
& =\left[\begin{array}{c}
\alpha_{1} I \\
\alpha_{2} I \\
\vdots \\
\alpha_{N} I
\end{array}\right]^{T}\left(\sum_{i=1}^{N} \alpha_{i}\left[\begin{array}{cccc}
\Upsilon_{1 i 1} & \Upsilon_{1 i 2} & \cdots & \Upsilon_{1 i N} \\
\Upsilon_{2 i 1} & \Upsilon_{2 N 2} & \cdots & \Upsilon_{2 i N} \\
\vdots & \vdots & \ddots & \vdots \\
Y_{N i 1} & Y_{N i 2} & \cdots & \Upsilon_{N i N}
\end{array}\right]\right)\left[\begin{array}{c}
\alpha_{1} I \\
\alpha_{2} I \\
\vdots \\
\alpha_{N} I
\end{array}\right] \leq 0 \text {. }
\end{aligned}
$$

Inequality (3.6) implies that (2.8) holds, so the theorem is proven. 
Remark 3.2. Theorem 3.1 provides a sufficient condition for the 2D system (2.1) with polytopic uncertainty (2.7) to be asymptotically stable in terms of LMI conditions. Note that if system (2.1) reduces to a 1D system with polytopic uncertainty, Theorem 3.1 coincides with the asymmetric stability for 1D systems considered in [19]. Therefore, Theorem 3.1 can be viewed as an extension of existing results on the asymptotic stability for 1D systems to the 2D case.

Remark 3.3. Theorem 3.1 contains both Lemmas 2.7 and 2.10 as particular cases. In the numerical examples, it will be shown that the condition proposed in Theorem 3.1 is the least conservative in comparison with the other four methods.

\section{Examples}

Example 4.1. The first example illustrates the main results for 2D uncertain system with three vertices. The system is parameterized by $r$ given by the triple $\left(r A_{1}, r A_{2}, r A_{3}\right)$ with the following vertices:

$$
\begin{aligned}
& A_{11}=\left[\begin{array}{ccc}
-0.0173 & -0.3258 & -0.2290 \\
0.0935 & -0.1020 & 0.0159 \\
-0.0076 & 0.3138 & 0.1993
\end{array}\right], \quad A_{21}=\left[\begin{array}{ccc}
-0.3338 & -0.0043 & -0.3715 \\
0.2128 & 0.5834 & 0.8134 \\
0.0676 & -0.2660 & -0.1896
\end{array}\right] \\
& A_{12}=\left[\begin{array}{ccc}
0.1253 & 0.3146 & 0.1192 \\
-0.0279 & -0.2348 & -0.1120 \\
-0.3263 & -0.1055 & 0.0595
\end{array}\right], \quad A_{22}=\left[\begin{array}{ccc}
0.0691 & -0.1205 & 0.1059 \\
0.1916 & -0.0150 & 0.6133 \\
-0.0397 & 0.0357 & -0.0941
\end{array}\right] \text {, } \\
& A_{13}=\left[\begin{array}{ccc}
0.4724 & 0.7253 & 0.6048 \\
0.1414 & 0.0115 & 0.1843 \\
-0.3777 & -0.5544 & -0.4840
\end{array}\right], \quad A_{23}=\left[\begin{array}{ccc}
-0.4187 & 0.5585 & -0.2581 \\
-0.1361 & -0.1769 & -0.2564 \\
0.2778 & 0.4486 & 0.5656
\end{array}\right] \text {, }
\end{aligned}
$$

where $A_{1}=\left[\begin{array}{ll}A_{11} & A_{21}\end{array}\right], A_{2}=\left[\begin{array}{ll}A_{12} & A_{22}\end{array}\right]$, and $A_{3}=\left[\begin{array}{ll}A_{13} & A_{23}\end{array}\right]$.

The robust stability of this system has been investigated by means of different lemmas cited above. The maximum value of $r$ such that the system $\left(r A_{1}, r A_{2}, r A_{3}\right)$ is stable, is only $r_{1}=$ 0.0691 using the QS approach (Lemma 2.7), but increases to $r_{2}=0.5745$ using Lemma 2.10, $r_{3}=$ 0.7521 using Lemma 2.11, $r_{4}=0.7551$ using Lemma 2.12, and $r_{5}=0.7552$ using Theorem 3.1. Thus, there is a significant improvement using the proposed approach.

Example 4.2. The objective is to check the stability of system (2.1) when the state matrix is within a polytope (2.8) with the following vertices:

$$
A_{11}=\left[\begin{array}{ccc}
-0.0304 & -0.5718 & -0.4019 \\
0.1641 & -0.1790 & 0.0279 \\
-0.0133 & 0.5508 & 0.3498
\end{array}\right], \quad A_{21}=\left[\begin{array}{ccc}
-0.5859 & -0.0075 & -0.6521 \\
0.3735 & 1.0240 & 1.4277 \\
0.1187 & -0.4669 & -0.3328
\end{array}\right]
$$




$$
\begin{aligned}
& A_{12}=\left[\begin{array}{ccc}
0.2199 & 0.5522 & 0.2092 \\
-0.0490 & -0.4121 & -0.1966 \\
-0.5727 & -0.1852 & 0.1044
\end{array}\right], \quad A_{22}=\left[\begin{array}{ccc}
0.1213 & -0.2115 & 0.1859 \\
0.3363 & -0.0263 & 1.0765 \\
-0.0697 & 0.0627 & -0.1652
\end{array}\right], \\
& A_{13}=\left[\begin{array}{ccc}
0.8292 & 1.2730 & 1.0615 \\
0.2482 & 0.0202 & 0.3235 \\
-0.6629 & -0.9731 & -0.8495
\end{array}\right], \quad A_{23}=\left[\begin{array}{ccc}
-0.7349 & 0.9803 & -0.4530 \\
-0.2389 & -0.3105 & -0.4500 \\
0.4876 & 0.7874 & 0.9927
\end{array}\right] \text {, }
\end{aligned}
$$

where $A_{1}=\left[\begin{array}{ll}A_{11} & A_{21}\end{array}\right], A_{2}=\left[\begin{array}{ll}A_{12} & A_{22}\end{array}\right]$, and $A_{3}=\left[\begin{array}{ll}A_{13} & A_{23}\end{array}\right]$.

This system has been selected so that it is possible to prove the robust stability using the proposed Theorem 3.1, but the previously proposed conditions in Lemmas 2.7, 2.10, 2.11, and 2.12 cannot prove the robust stability, as the corresponding LMIs are not feasible. The vertices of the parameter-dependent Lyapunov matrices that fulfill the conditions of Theorem 3.1 are the following:

$$
\begin{aligned}
& P_{1}=\left[\begin{array}{ccc}
0.2478 & 0 & 0 \\
0 & 0.2478 & 0 \\
0 & 0 & 0.2478
\end{array}\right], \quad P_{2}=\left[\begin{array}{ccc}
0.2478 & 0 & 0 \\
0 & 0.2478 & 0 \\
0 & 0 & 0.2478
\end{array}\right], \\
& P_{3}=\left[\begin{array}{ccc}
0.2478 & 0 & 0 \\
0 & 0.2478 & 0 \\
0 & 0 & 0.2478
\end{array}\right], \quad Q_{1}=\left[\begin{array}{ccc}
0.2671 & 0.1629 & 0.4052 \\
0.1629 & 0.3489 & 0.5172 \\
0.4052 & 0.5172 & 1.0891
\end{array}\right] \text {, } \\
& Q_{2}=\left[\begin{array}{lll}
0.2224 & 0.0242 & 0.2778 \\
0.0242 & 0.0465 & 0.0027 \\
0.2778 & 0.0027 & 0.6236
\end{array}\right], \quad Q_{3}=\left[\begin{array}{ccc}
0.0744 & -0.1024 & 0.0442 \\
-0.1024 & 0.7262 & 0.2207 \\
0.0442 & 0.2207 & 0.1619
\end{array}\right] \text {, } \\
& R_{1}=\left[\begin{array}{ccc}
0.0201 & 0.0095 & 0.0263 \\
0.0095 & 0.0064 & 0.0123 \\
0.0263 & 0.0123 & 0.0397
\end{array}\right], \quad R_{2}=10^{-7} \times\left[\begin{array}{ccc}
0.3724 & 0.1235 & 0.1291 \\
0.1235 & 0.6149 & -0.2036 \\
0.1291 & -0.2036 & 0.5666
\end{array}\right] \text {, } \\
& R_{3}=10^{-6} \times\left[\begin{array}{ccc}
0.0141 & -0.0015 & 0.0098 \\
-0.0015 & 0.1565 & -0.0538 \\
0.0098 & -0.0538 & 0.0608
\end{array}\right] \text {. }
\end{aligned}
$$

\subsection{Numerical evaluation}

Finally, a numerical evaluation procedure is considered to check the improvements obtained with the proposed method when testing the stability of plants randomly generated. Recall that the kind of systems we are dealing with is characterized by its order $(n)$, and the number 
Table 1: Number of robustly stable systems correctly confirmed by the different methods for $2 \leq n \leq 4$, $2 \leq N \leq 3$ (from a maximum of 1000 systems for each $\{n, N\}$ ).

\begin{tabular}{ccccccc}
\hline$n$ & $N$ & Lemma 2.7 & Lemma 2.10 & Lemma 2.11 & Lemma 2.12 & Theorem 3.1 \\
\hline 2 & 2 & 582 & 860 & 928 & 940 & 945 \\
& 3 & 289 & 691 & 817 & 831 & 834 \\
\hline 3 & 2 & 213 & 709 & 827 & 828 & 844 \\
& 3 & 25 & 481 & 656 & 674 & 683 \\
\hline \multirow{2}{*}{4} & 2 & 93 & 445 & 608 & 625 & 747 \\
& 3 & 2 & 234 & 429 & 439 & 456 \\
\hline
\end{tabular}

of vertices in its polytopic description $(N)$. Thus, 1000 systems were randomly generated for each pair of values $\{n, N\}$ in the ranges $2 \leq n \leq 4$, and $2 \leq N \leq 3$, giving a total of 6000 stable polytopes. Each of these polytopes was evaluated using the different methods to check if the conditions successfully confirmed the robust stability. The results are given in Table 1, which exhibits the number of success of the different methods, which gives a measure of their performance and conservativeness. It can be seen that the tests given by Theorem 3.1 prove to be significantly less conservative than the original QS test (Lemma 2.7) and the modified versions derived in Lemmas 2.10, 2.11 and 2.12. Of course the computational cost increases, being the most computationally demanding the test corresponding to Theorem 3.1, but always within acceptable computational times.

\section{Conclusions}

This paper has proposed conditions to determine the robust stability of 2D linear discrete-time systems described by Fornasini-Marchesini second models, under polytopic uncertainty. The conditions are formulated in terms of sets of LMIs described only in terms of the vertices of the uncertainty domain. Several examples have been presented that illustrate the results, showing the feasibility of the proposed approaches.

\section{Acknowledgment}

This work has been funded by AECI research projects A/5422/06, A/7882/07, and projects DPI2004-07444-C04-02 and DPI2007-66718-C04-02.

\section{References}

[1] B. R. Barmish, "Necessary and sufficient conditions for quadratic stabilizability of an uncertain system," Journal of Optimization Theory and Applications, vol. 46, no. 4, pp. 399-408, 1985.

[2] J. Bernussou, P. L. D. Peres, and J. C. Geromel, "A linear programming oriented procedure for quadratic stabilization of uncertain systems," Systems $\mathcal{E}$ Control Letters, vol. 13, no. 1, pp. 65-72, 1989.

[3] S. Boyd, L. El Ghaoui, E. Feron, and V. Balakrishnan, Linear Matrix Inequalities in System and Control Theory, vol. 15 of SIAM Studies in Applied Mathematics, SIAM, Philadelphia, Pa, USA, 1994.

[4] H. R. Karimi, "Robust stabilization with $H_{\infty}$ performance for a class of linear parameter-dependent systems," Mathematical Problems in Engineering, vol. 2006, Article ID 59867, 15 pages, 2006.

[5] M. C. de Oliveira, J. C. Geromel, and L. H. Su, "LMI characterization of structural and robust stability: the discrete-time case," Linear Algebra and Its Applications, vol. 296, no. 1-3, pp. 27-38, 1999. 
[6] E. Feron, P. Apkarian, and P. Gahinet, "Analysis and synthesis of robust control systems via parameter-dependent Lyapunov functions," IEEE Transactions on Automatic Control, vol. 41, no. 7, pp. 1041-1046, 1996.

[7] V. J. S. Leite and P. L. D. Peres, "An improved LMI condition for robust D-stability of uncertain polytopic systems," IEEE Transactions on Automatic Control, vol. 48, no. 3, pp. 500-504, 2003.

[8] D. C. W. Ramos and P. L. D. Peres, "A less conservative LMI condition for the robust stability of discrete-time uncertain systems," Systems E Control Letters, vol. 43, no. 5, pp. 371-378, 2001.

[9] A. Rantzer and M. Johansson, "Piecewise linear quadratic optimal control," IEEE Transactions on Automatic Control, vol. 45, no. 4, pp. 629-637, 2000.

[10] L. Xie, S. Shishkin, and M. Fu, "Piecewise Lyapunov functions for robust stability of linear timevarying systems," Systems \& Control Letters, vol. 31, no. 3, pp. 165-171, 1997.

[11] D. Peaucelle, D. Arzelier, O. Bachelier, and J. Bernussou, "A new robust D-stability condition for real convex polytopic uncertainty," Systems \& Control Letters, vol. 40, no. 1, pp. 21-30, 2000.

[12] T. Kaczorek, Two-Dimensional Linear Systems, vol. 68 of Lecture Notes in Control and Information Sciences, Springer, Berlin, Germany, 1985.

[13] H. D. Tuan, P. Apkarian, T. Q. Nguyen, and T. Narikiyo, "Robust mixed $\mathscr{\ell}_{2} / \mathscr{\ell}_{\infty}$ filtering of 2-D systems," IEEE Transactions on Signal Processing, vol. 50, no. 7, pp. 1759-1771, 2002.

[14] T. Hinamoto, "2-D Lyapunov equation and filter design based on the Fornasini-Marchesini second model," IEEE Transactions on Circuits and Systems I, vol. 40, no. 2, pp. 102-109, 1993.

[15] T. Ooba, "On stability analysis of 2-D systems based on 2-D Lyapunov matrix inequalities," IEEE Transactions on Circuits and Systems I, vol. 47, no. 8, pp. 1263-1265, 2000.

[16] E. Fornasini and G. Marchesini, "Stability analysis of 2-D systems," IEEE Transactions on Circuits and Systems, vol. 27, no. 12, pp. 1210-1217, 1980.

[17] A. Hmamed, M. Alfidi, A. Benzaouia, and F. Tadeo, "Robust stabilization under linear fractional parametric uncertainties of 2-D systems with Roesser models," International Journal on Sciences and Techniques of Automatic Control, 2008.

[18] A. Hmamed, M. Alfidi, A. Benzaouia, and F. Tadeo, "LMI conditions for robust stability of 2-D uncertain discrete-time linear systems," in Proceedings of the Conference on Systems and Control (CSC '07), Marrakech, Morocco, May 2007.

[19] S.-W. Kau, Y.-S. Liu, L. Hong, C.-H. Lee, C.-H. Fang, and L. Lee, “A new LMI condition for robust stability of discrete-time uncertain systems," Systems $\mathcal{E}$ Control Letters, vol. 54, no. 12, pp. 1195-1203, 2005. 


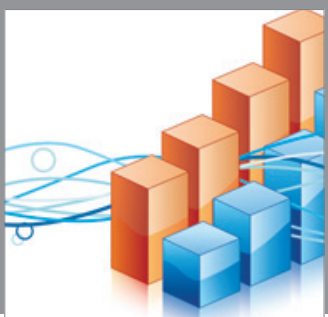

Advances in

Operations Research

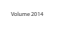

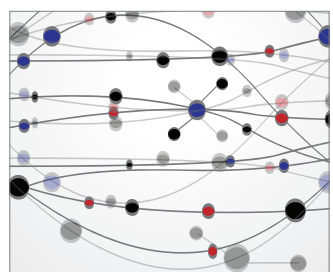

\section{The Scientific} World Journal
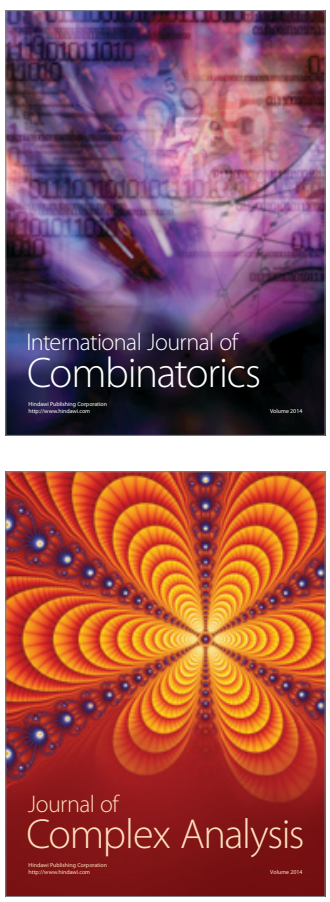

International Journal of

Mathematics and

Mathematical

Sciences
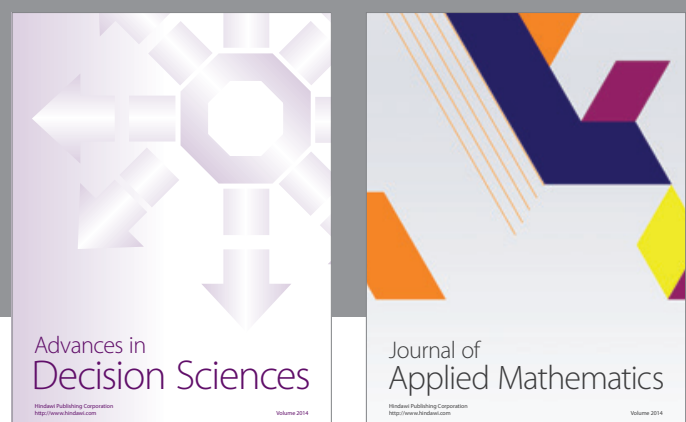

Journal of

Applied Mathematics
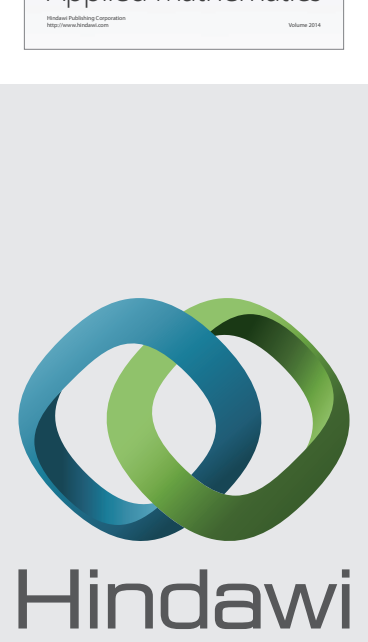

Submit your manuscripts at http://www.hindawi.com
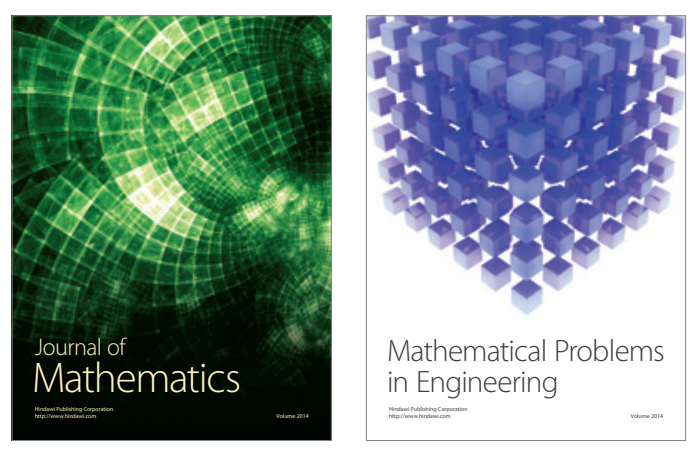

Mathematical Problems in Engineering
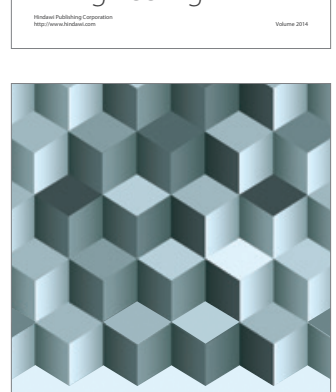

Journal of

Function Spaces
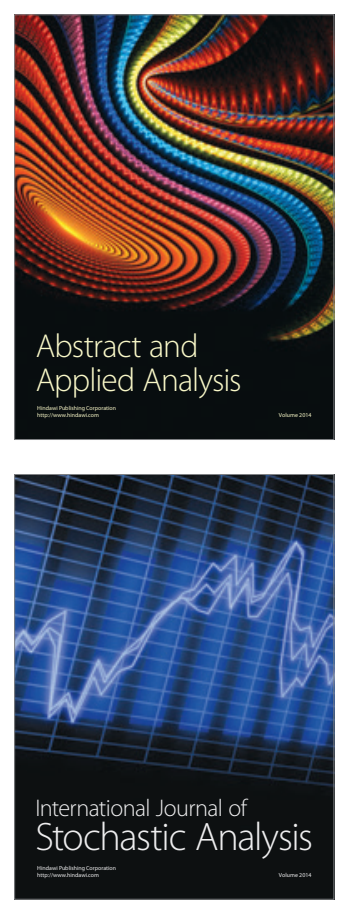

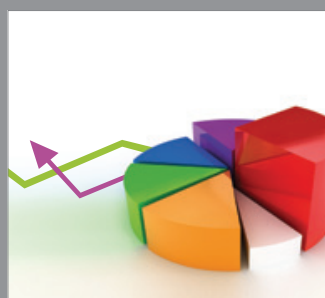

ournal of

Probability and Statistics

Promensencen
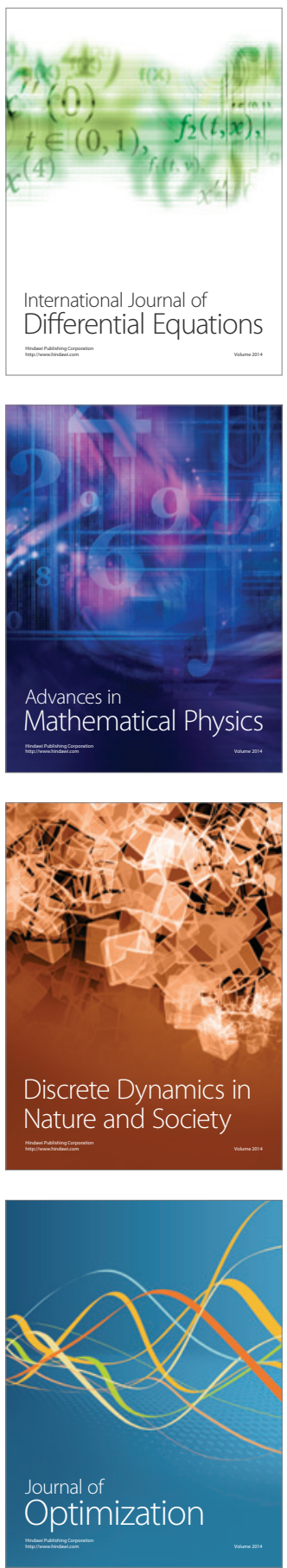